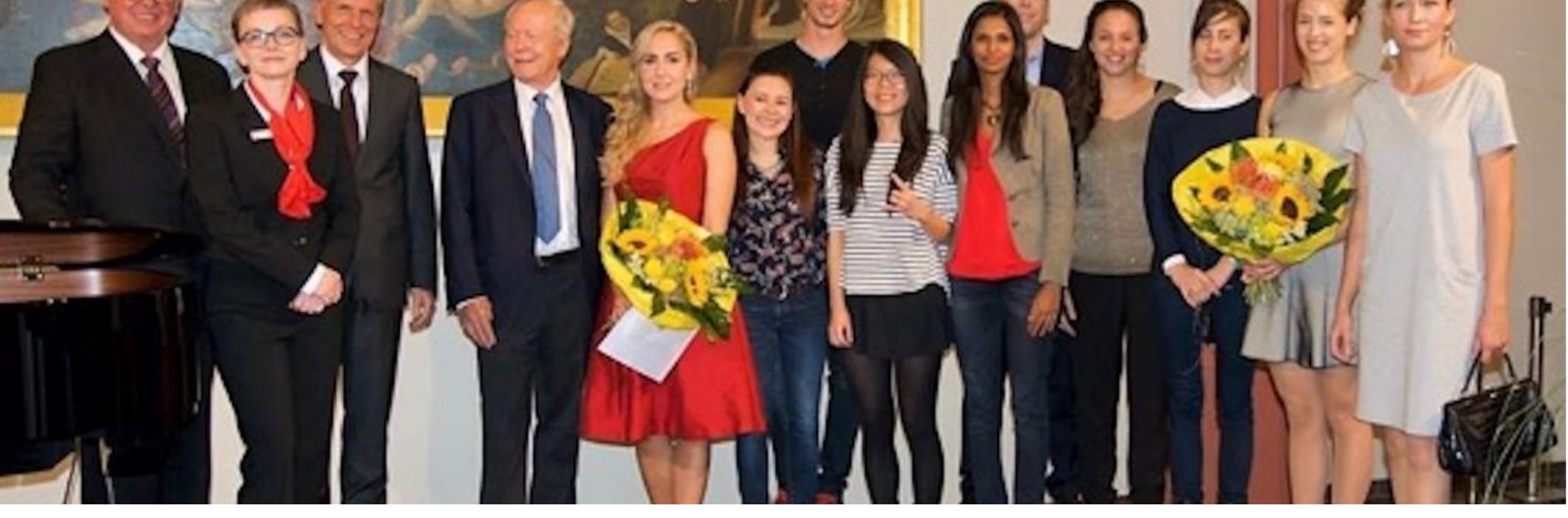

ART - THE MOTHER OF ALL DISCOVERIES.

Authors: $\quad$ Yaneth Ortiz

Submitted:

28. October 2017

Published:

Volume:

29. October 2017

Issue:

Affiliation:

Keywords:

DOI:

4

5

JOSHA

Kim Strebel, IASHA event, Sparkasse, Soprano.

10.17160/josha.4.5.347

Journal of Science,

Humanities and Arts 


\section{ART - THE MOTHER OF ALL DISCOVERIES}

On September 13, 2017, the Journal of Science, Humanities and Arts editorial board, collaborators and close friends enjoyed a wonderful artistic night with the amazing performance by the soprano Kim-Lillian Strebel and pianist Daniel Carter, delightfully accompanied by the moving poetry of the writer Zazie-Charlotte Pfeiffer. The beautiful Meckelhalle in Freiburg was the perfect scenario for the event, made possible thanks to the cooperation of the International Academy of Science, Humanities and Arts (IASHA e.V.), the BioThera-Stiftung Foundation Freiburg and the Sparkasse.

The close connection between artistic and scientific creativity has become increasingly clear in recent years. This exchange of ideas and creative concepts between scientists and artists, is the concern of the International Academy of Science, Humanities and Arts (IASHA
e.V.), publisher of the open access, multidisciplinary, multilingual journal JOSHA, a platform publishing monographs, manuscripts, books and articles from all disciplines for readers all around the world. Containing works from Nobel Prize winners to young scientists and artists.

The non-profit BioThera-Stiftung Foundation supports projects in cancer research and young Scientist and artist through scholarship awards and cooperation projects for students, doctors, scientists and artists and much more.

IASHA and JOSHA appreciate the collaboration offered by Sparkasse and BioThera Foundation and the support received by all the assistants to the event and hope to meet again in future events. 


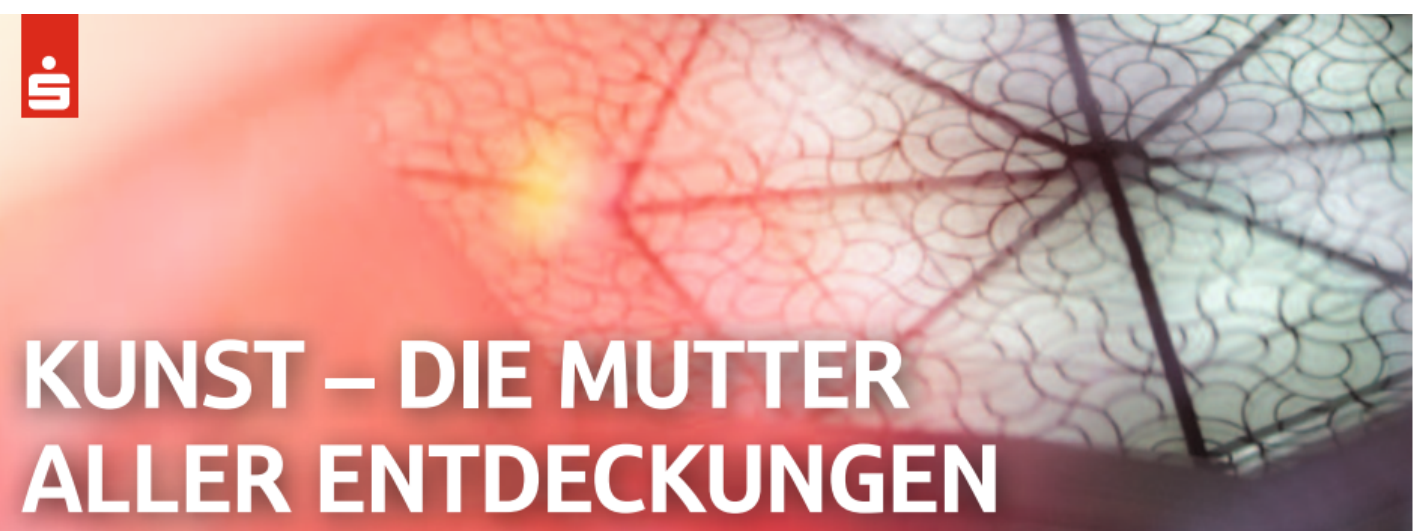

Ein Liederabend mit der Sopranistin Kim-Lillian Strebel / Eine Kooperation mit der International Academy of Science, Humanities and Arts (IASHA e.V.) und der Biothera-Stiftung Freiburg

13.9.2017 | 19 Uhr | Meckelhalle Freiburg, Sparkassen-FinanzZentrum, Eingang Franziskanerstraße

Der aktuelle Anlass des Konzerts von Kim-Lillian Strebel ist die Verleihung eines einjährigen Stipendiums 2016/2017 zur Förderung der jungen Künstlerin durch die "International Academy of Sciences, Humanities and Arts (IASHA e.V.)“ mit Unterstützung der Biothera-Stiftung Freiburg.

Die britische Sopranistin Kim-Lillian Strebel war bis 2017 Ensemblemitglied des Theaters Freiburg, wo sie u.a. Partien wie Micaëla / CARMEN, Fiordiligi / COSì FAN TUTTE, Isaura in einer Inszenierung Calixto Bieitos von Verdis JERUSALEM unter Stabführung von Fabrice Bollon und nicht zuletzt die Titelpartie in Cendrillon interpretierte. In diesem Jahr ist Kim-Lillian Strebel auf Amerikatournee, u.a. in Atlanta mit dem Atlanta Symphonie Orchester und als Pamina in Barrie Koskys DIE ZAUBERFLÖTE an der Cincinatti Opera. Sie wuchs in der Schweiz auf. Bereits von früher Kindheit an widmete sie sich dem Tanz. Später ging sie nach London, wo sie bei Anthony Rolfe-Johnson Gesangsunterricht erhielt. Später setzte sie ihre Studien mit einem Stipendium am Royal Conservatoire of Scotland und als Privatschülerin bei Dame Kiri Te Kanawa und Ryland Davies fort.

Der enge Zusammenhang zwischen künstlerischer und wissenschaftlicher Kreativität ist in den letzten Jahren immer deutlicher geworden. Diesen wechselseitigen Austausch von Ideen und kreativen Konzepten zwischen Wissenschaftlern und Künstlern zu fördern, ist das Anliegen der ${ }_{n}$ International Academy of Science, Humanities and Arts“ (IASHA e.V.), Freiburg, der von ihr herausgegeben „open access“ multidisziplinären, mehrsprachigen Zeitschrift JOSHA und der BioThera-Stiftung, Freiburg. IASHA E.V widmet sich der Förderung der Wissenschaft und Kunst. Das "open access" Online-Journal des Vereins ist das "Journal of Sciences, Humanities and Arts", www.josha-journal.org. Es dient als Austauschplattform für Publikationen von Nobelpreisträgern, jungen Wissenschaftlern und Künstlern. Die Gemeinnützige Biothera-Stiftung fördert Projekte zur Krebsforschung sowie den wissenschaftlichen und künstlerischen Nachwuchs durch Stipendien Preise und Kooperationsprojekte für Studenten, Ärzte, Wissenschaftler und Künstler und vieles mehr.

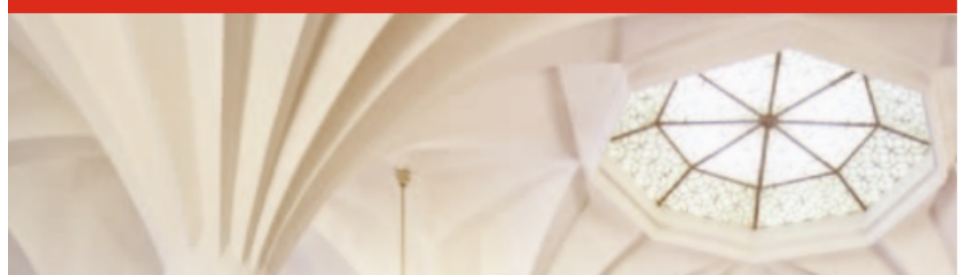

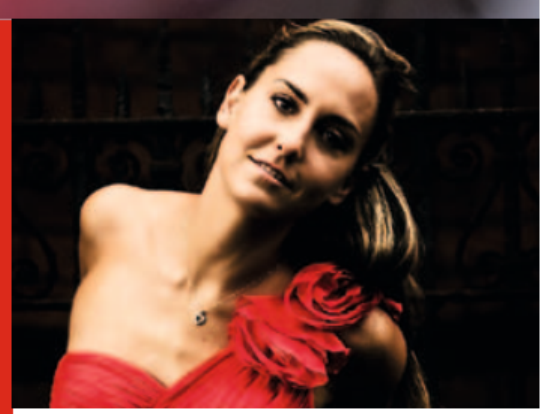

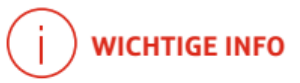

Um Rückmeldung bitten wir auf beiliegendem Antwortbogen bis 4. September 2017. Teilnahme nur solange Plätze vorhanden!

\section{(I) PROGRAMM}

Begrüßung

Marcel Thimm

Vorstandsvorsitzender

\section{Grußwort und Einführung}

Prof. Dr. Dr. h.c. mult. Roland Mertelsmann Stellvertretender Vorsitzender IASHA e.V. Vorstand der Biothera-Stiftung

Felicitas Holzer, M.Sc., MPhil Mitglied IASHA e.V.

Stipendiatin der Biothera-Stiftung

\section{Konzert:}

Kunst - Die Mutter aller Entdeckungen

Kim-Lillian Strebel, Sopranistin Daniel Carter, Pianist

Poetry: Die Nacht - eine Kurzgeschichte Zazie-Charlotte Pfeiffer, cand.med., Poetin

\section{Moderation}

Prof.Dr. Dr. h.c. mult. Roland Mertelsmann

Im Anschluss laden wir Sie zu einem Imbiss mit Umtrunk ein. 


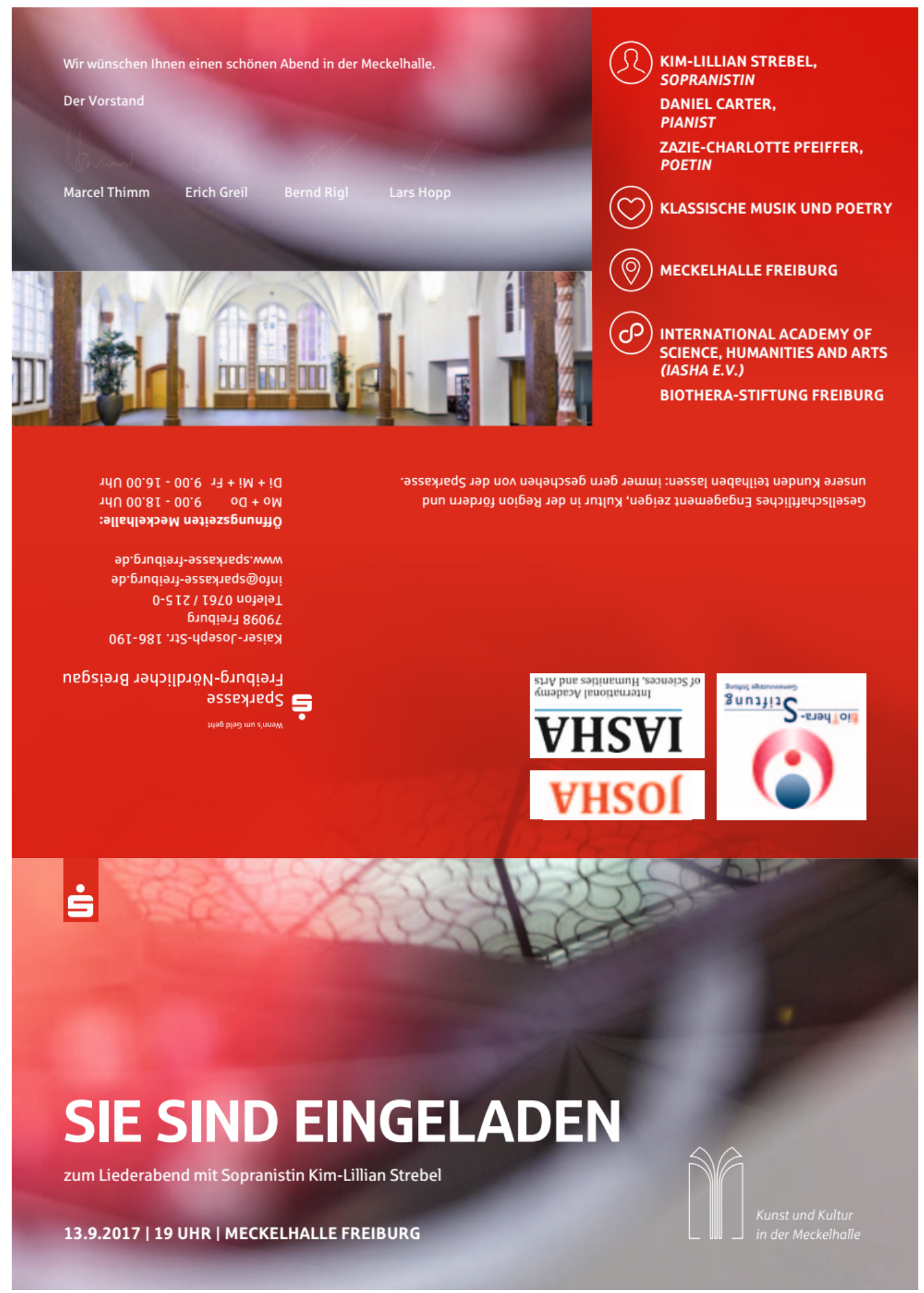



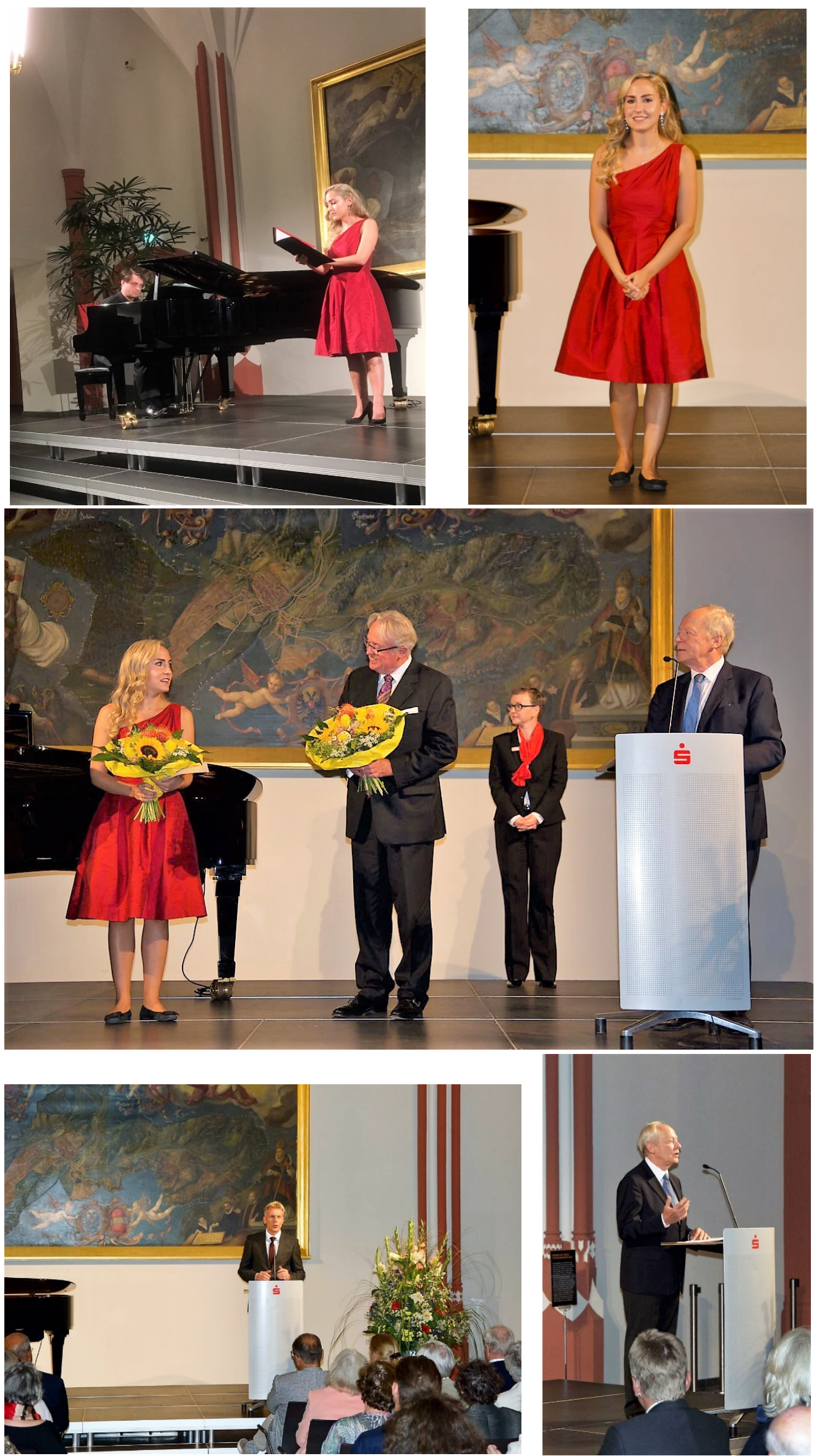

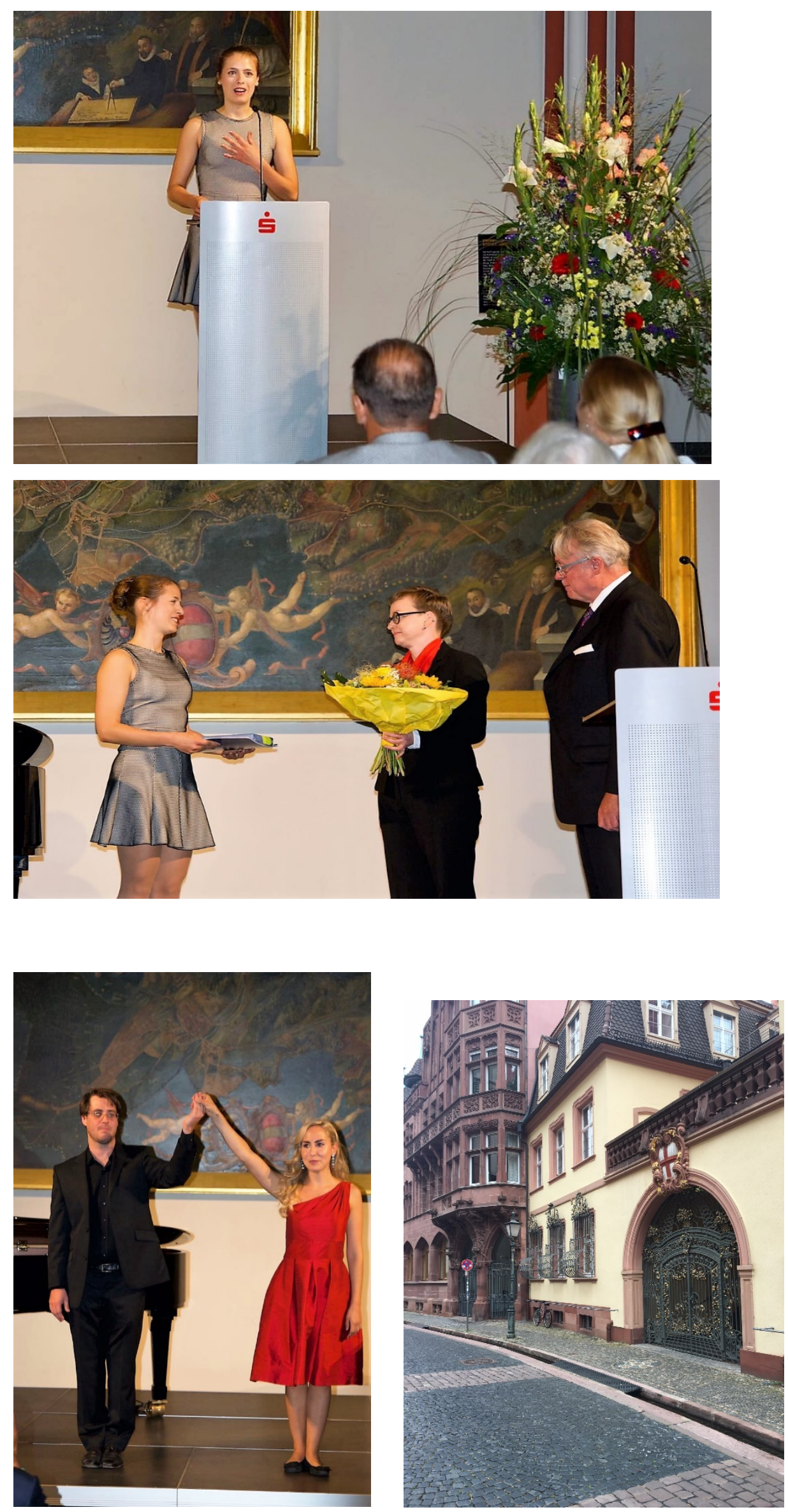